\title{
SEARCH FOR ANOMALOUS MULTIPHOTON PRODUCTION AT 100-300 GeV
}

\author{
D.L. BURKE, H.R. GUSTAFSON, L.W. JONES and M.J. LONGO \\ University of Michigan, Ann Arbor, Michigan 48104, USA
}

\begin{abstract}
We have carried out a search for anomalous multiphoton production in neutron- $\mathrm{CH}_{2}$ collisions at Fermilab. Both anomalous $\gamma$ events as might be produced in the annihilation of a magnetic monopole pair, as well as events with smaller opening angles, such as those observed in cosmic ray emulsions by Schein et al. and others were sought. No evidence for either type of event was found. An upper limit $\sim 2.7 \mu \mathrm{b}$ is placed on the production cross section for "Schein" events or $\sim 10^{-2}$ that deduced from the cosmic ray data.
\end{abstract}

Numerous searches for free magnetic monopoles have yielded negative results ${ }^{\ddagger 1}$ and have set extremely small upper limits on monopole production cross sections [2-4]. It has been suggested, however, that due to the long-range strong magnetic force between a pole and an antipole the production of a pole pair would be followed by their annihilation into a large number of photons. The number of photons produced depends primarily on the magnetic pole strength $g$, which must be quantized so that

$e g / h c=n / 2, \quad n=1,2,3 \ldots$.

Ruderman and Zwanziger [5] have suggested that the annihilation of magnetic monopole pairs produced by high-energy photons provides a possible explanation for several anomalous jets of pure photons observed in emulsions exposed to cosmic rays [6-9]. These events, first observed by Schein et al. [6], have several peculiar properties, notably their high degree of collimation, lack of charged particles, and the large number of photons produced. Their properties are summarized in table $1[10]$. Using the given parameters of the exposures, we can estimate the cross sections for Schein event production by cosmic photons as well as by neutrons produced in the atmosphere. For production by photons of $E \gtrsim 100 \mathrm{GeV}$ we obtain a cross section $\sim 3$ $\mathrm{mb}$ per nucleon. The cross section for production by

\footnotetext{
Work supported by the U.S. National Science Foundation.

$\neq 1$ The observation of an anomalous cosmic ray particle has been recently reported in the literature [1]. The authors interpret these data as the detection of a magnetic monopole. Alternate interpretations have been offered elsewhere.
}

Table 1

Properties of anomalous multiphoton events observed in emulsions

\begin{tabular}{llll}
\hline & $\begin{array}{l}\text { Estimated } \\
\text { energy } \\
\text { Reference }\end{array}$ & $\begin{array}{l}\text { Opening } \\
\text { angle } \\
\text { (radians) }\end{array}$ & $\begin{array}{l}\text { No. of pairs } \\
\text { formed in } 1.22 L \\
\text { following } \\
\text { first pair a) }\end{array}$ \\
\hline Schein et al. [6] & $\sim 90$ & $\sim 0.5$ & 18 \\
& & $\times 10^{-3}$ & \\
DeBenedetti et al. [7] & & & \\
(1) & $>40$ & $\sim 10^{-3}$ & 14 \\
(2) & $>60$ & $\sim 10^{-3}$ & $9(24$ in $1.6 L)$ \\
Silva et al. [9] & $>100$ & $\sim 10^{-4}$ & $21($ in $1.14 L)$ \\
Koshiba et al. [8] & $\sim 100$ & $\sim 10^{-3}$ & 16
\end{tabular}

a) $L \equiv$ radiation length $=2.9 \mathrm{~cm}$ in emulsion

neutrons is $\sim 0.3 \mathrm{mb}$ per nucleon [11].

The experimental arrangement used in this search is shown in fig. 1. A neutron beam [12] with a maximum energy of $300 \mathrm{GeV}$ (most probable energy $\approx 240 \mathrm{GeV}$ ) was incident on a $6.8 \mathrm{~cm}$ thick $\mathrm{CH}_{2}$ target

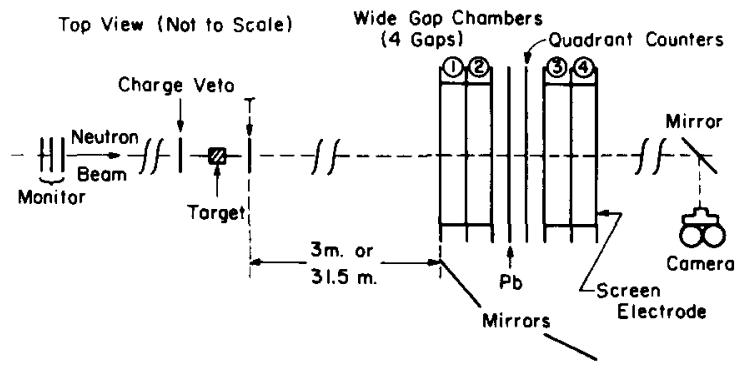

Fig. 1. Experimental apparatus. 
Charged particles and photons were detected by an optical spark chamber array, consisting of four $15 \mathrm{~cm}$ gaps on $1 \mathrm{~m}^{2}$ area [13]. A lead converter $6.4 \mathrm{~mm}$ thick between the 2 nd and 3 rd gaps covered the active area of the chambers except for a $2.5 \mathrm{~cm}$ diameter hole centered on the beam. Immediately behind the lead was a set of trigger counters whose total thickness was $3.8 \mathrm{~cm}$.

One side view of all four gaps was photographed as well as a $90^{\circ}$ stereo view of the central $50 \mathrm{~cm}$ of the first gap. On the same $35 \mathrm{~mm}$ film frame, a "beam's eye" view of the last gap was obtained through the screen electrode as shown in fig. 1.

The efficiency of the chambers as measured by the number of tracks lost in going from one chamber to the other was $>95 \%$ (integrated over the angular range $0^{\circ}$ to $25^{\circ}$ ) and was shown to be independent of the number of tracks to $\approx 30$ tracks. As many as 175 tracks have been observed in the last gap.

The trigger counters were used to select events of potential interest. A pulse height cut on the target counter (T) was employed to limit the number of charged particles produced in the target to typically three or four. In addition, four counters, each covering one quadrant of the active chamber area, were used to require that a large number $(\sim 10)$ of charged particles be produced in the lead converter. Two triggers were formed by placing the target counter in coincidence with each of the four quadrant counters (trigger $A$ ) or with the sum of the quadrant counters (trigger $B$ ).

In addition to these selective triggers, data were also taken with an inclusive trigger which required at least one charged particle in the $T$ counter and at least one of the quadrant counters. This trigger was used to normalize the selective triggers to the $\mathrm{CH}_{2}$ inelastic cross section. We estimate this normalization to be accurate to $\pm 5 \%$.

A summary of the data taken is given in table 2 . With the target at the $3 \mathrm{~m}$ position, triggers $A$ and $B$ were used to search for events which produce an anomalous number of photons more or less isotropically in the nucleon-nucleon CMS. At the $31.5 \mathrm{~m}$ target position, both triggers were used to search for anomalous events with photons collimated to $<16 \mathrm{mr}$. In addition, trigger $B$ was used at this position to look for offaxis jets of photons with opening angles $\leqslant 1 \mathrm{mr}$.

We define a "neutral prong" to be a track that ap-
Table 2

Summary of data.

\begin{tabular}{rlll}
\hline $\begin{array}{l}\text { Target } \\
\text { position }\end{array}$ & Trig. & $\begin{array}{l}\text { Acceptance } \\
\text { angle }\end{array}$ & $\begin{array}{l}\text { No. of } \\
\text { trigs. }\end{array}$ \\
\hline $3 \mathrm{~m}$ & $\mathrm{~A}$ & $90^{\circ} \mathrm{N}-\mathrm{N} \mathrm{CMS}$ & 6190 \\
$3 \mathrm{~m}$ & $\mathrm{~B}$ & $90^{\circ} \mathrm{N}-\mathrm{N} \mathrm{CMS}$ & 3814 \\
$31.5 \mathrm{~m}$ & $\mathrm{~A}$ & $\pm 16 \mathrm{mr}$ (lab) & 3129 \\
$31.5 \mathrm{~m}$ & $\mathrm{~B}$ & $\pm 16 \mathrm{mr}$ (lab) & 3907 \\
\hline
\end{tabular}

pears in gaps 3 or 4 for which there is no associated track in gaps 1 and 2 (see fig. 1). The scanning procedure consisted of counting the number of neutral prongs in each event. Each event for which this count exceeded a particular value (typically 25 to 30 ) was then rescanned by a physicist, noting the number of vertices produced in the lead by neutral particles which could be resolved by eye. Since all of the photons in a $1 \mathrm{mr}$ jet would strike the lead within a 3.3 $\mathrm{cm}$ diameter circle, other methods had to be used to search for Schein events (see below).

The results for the $3 \mathrm{~m}$ target position are summarized in table 3. As a guide to the number of $\gamma$ 's "expected" from a monopole pair annihilation we use a calculation made by Newmeyer and Trefil [14]. From a thermodynamic model they estimate $\bar{N}_{\gamma}$, the average number of $\gamma$ 's, to be $32,59,91$ for $n=2,3,4$ resp. (eq. 1). The probability of a photon converting in the lead is $\approx 0.60$, and if we assume the photons are emitted approx. isotropically in the N-N CMS, about half the photons strike our detector. Thus, for

Table 3

$\bar{N}_{\mathrm{V}}$ is the average number of vertices expected for a given photon multiplicity $\vec{N}_{\gamma}$. The number of events observed with $\geqslant \bar{N}_{\mathrm{V}}$ vertices is shown along with the corresponding cross section limits per nucleon.

\begin{tabular}{|c|c|c|c|c|}
\hline \multirow[b]{2}{*}{ Trig. } & \multirow[b]{2}{*}{$\bar{N}_{\gamma}$} & \multicolumn{2}{|c|}{ Expected events } & \multirow[b]{2}{*}{$\begin{array}{l}\sigma(90 \%) \\
\mu \mathrm{b}\end{array}$} \\
\hline & & $\overline{N_{\mathrm{v}}}$ & $\begin{array}{l}\geqslant \bar{N}_{\mathrm{V}} \\
\text { verts }\end{array}$ & \\
\hline \multirow{3}{*}{ A } & 32 & 10 & $22^{a}$ & $\leqslant 13.2$ \\
\hline & 59 & 15 & 0 & $\leqslant 0.7$ \\
\hline & 91 & 27 & 0 & $\leqslant 0.5$ \\
\hline \multirow{3}{*}{ B } & 32 & 10 & $6^{a}$ & $\leqslant 9.2$ \\
\hline & 59 & 15 & 0 & $\leqslant 2.3$ \\
\hline & 91 & 27 & 0 & $\leqslant 2.6$ \\
\hline
\end{tabular}

a Less than 6 charged prongs were required in gaps 1 and 2 . 
a given $\bar{N}_{\gamma}$ we expect $\approx 0.3 \bar{N}_{\gamma}$ vertices in the detector. Table 3 lists, for each value of $\bar{N}_{\gamma}$, the expected average number of vertices $\bar{N}_{\mathrm{v}}$, the number of events observed with $\geqslant \bar{N}_{\mathrm{v}}$ vertices, and the corresponding limits on the cross section for producing anomalous events. No events were observed with $>14$ vertices.

A Monte Carlo simulation based on extrapolations to large multiplicities of recent FNAL bubble chamber data has been written to estimate the background expected from known processes. Secondary neutrons and $\gamma$ 's from $\pi^{\circ}$ 's were traced through the apparatus applying the proper detection efficiencies. Neutroncarbon nucleus collisions were simulated using a leading particle cascade model [11]. The 28 events observed with $\geqslant 10$ vertices are consistent with the results of this simulation without involving anomalous production.

The cross section limits in table 3 are per nucleon and have been corrected for our triggering efficiency ( $8 \%$ to $65 \%$ depending on multiplicity) and the probability of an anomalous event meeting our scanning and event selection criteria ( $13 \%$ to $100 \%$ ). These corrections are somewhat model dependent. We have assumed a final state consisting of the two initial hadrons and the number of photons quoted in the tables. The corrections are then estimated taking account of photon conversion probabilities and allowing $40 \%$ probability that the leading hadron becomes a proton.

The $31.5 \mathrm{~m}$ target position was used to look for highly collimated events of the Schein type; such events would lie within the $\pm 16 \mathrm{mr}$ acceptance of the detector. The cosmic ray events (table 1 ) had 15-20 vertices in the first $1.22 \mathrm{rad}$. lengths ${ }^{\ddagger 2}$. For comparison, in table 4 we list the number of events observed with $\geqslant 7,9$ or 12 vertices. The largest number of vertices observed was 9 and again the data were well fitted by the Monte Carlo without anomalous production. The cross section limits in table 4 include corrections for triggering and detection efficiency.

Trigger B in which all four quadrant counters were summed was used to look for off-axis Schein jets. Since we are studying hadron induced reactions, then it is reasonable to expect that any photon jet would

$\neq 2$ The event no. 2 of ref. [7] has a peculiar spatial distribution. It is reasonable to assume that the number of photons reaching our detector from an event of this type would produce $\sim 20$ pairs.
Table 4

The number of events observed with $\geqslant N_{\mathrm{v}}$ vertices and cross section limits for the $31.5 \mathrm{~m}$ target position.

\begin{tabular}{lccl}
\hline Trig. & $N_{\mathrm{V}}$ & $\begin{array}{l}\text { No. events } \\
\geqslant N_{\mathrm{V}} \text { verts. }\end{array}$ & $\begin{array}{l}\sigma(90 \%) \\
\mu \mathrm{b}\end{array}$ \\
\hline A & 7 & 10 & $\leqslant 3.7$ \\
& 9 & 1 & $\leqslant 0.2$ \\
& 12 & 0 & $\leqslant 0.2$ \\
B & 7 & 0 & $\leqslant 8.5$ \\
& 9 & 0 & $\leqslant 3.3$ \\
& 12 & 0 & $\leqslant 3.0$ \\
\hline
\end{tabular}

be produced with $\left\langle p_{\perp}\right\rangle \approx 0.35 \mathrm{GeV} / c$. Only if the process occurs nearly at threshold would the transverse momentum be significantly less. Taking the total energy in the jet $\approx 100 \mathrm{GeV}$ (as observed in the cosmic ray events) we obtain a lateral displacement at the detector $\approx 10 \mathrm{~cm}$. Thus the $2.5 \mathrm{~cm}$ diameter hole in the lead converter is not a restriction to our search.

The limits in table 4 are based on vertex counting. Photons from Schein events whose opening angles were $\lesssim 1 \mathrm{mr}$ would appear in the chambers as a single unresolved vertex with 30 or more prongs. It was found that of the 75 events with $\geqslant 30$ prongs initiated by a neutral particle, 58 had prongs going backward into gaps 1 and 2. It is unlikely that photon will produce any backward traveling particles in $1.22 \mathrm{rad}$. lengths and we assume these events are due to neutron interactions in the lead. For the remaining events the angular distribution of secondary tracks which a Schein event should produce was considered. The typical photon energy is about $2 \mathrm{GeV}$ [6-9]. A Schein event producing 15-20 pairs would be expected to produce at least 10-15 tracks within $31 \mathrm{mr}$ in our detector (i.e., the higher energy electron from each pair has a $70 \%$ chance of remaining within an angle $\leqslant 31 \mathrm{mr}$ ).

We have measured and reconstructed the 17 events in question. The largest number of prongs produced within $31 \mathrm{mr}$ of any point on the "vertex" was found to be 8 . We conclude that these events are due to neutrons and at a $90 \%$ C.L. place an upper limit on the cross section for Schein events of $\sigma(90 \%) \leqslant 2.7 \mu \mathrm{b}$ per nucleon after correcting for the detection efficiency.

The $31.5 \mathrm{~m}$ of air ahead of our detector and the target total $\approx 0.2 \mathrm{rad}$. length. Thus there is a high probability that upon reaching the detector the photons from a Schein event would be accompanied by a 
pari (or more) of charged particles within the cone of the jet ${ }^{\ddagger 3}$. To search for photon jets accompanied by 2 or more charged particles, the film was scanned for events containing 2 or more tracks in gaps 1 and 2 within $3 \mathrm{~cm}$ of each other as determined by all views. No constraint was placed on the number of additional charged particles outside of the assumed jet except that imposed by the trigger. While Schein events should produce 30 to 40 prongs, we see no events which produced more than 29 . The observed distribution of prongs produced in the lead by these particles is well fitted by a Monte Carlo simulation which assumes that the charged particles are electrons unaccompanied by any photons. We again conclude that no Schein events are observed and place an upper limit on the cross section of $\sigma(90 \%) \leqslant 7.9 \mu \mathrm{b}$ per nucleon.

In conclusion we have seen no evidence for the production and subsequent annihilation of magnetic monopole pairs into photons. The cross section limits are $\sim 2 \mu \mathrm{b}$. (See tables 3 and 4 ). No evidence for Schein events has been seen and an upper limit of 2.7 $\mu \mathrm{b}$ has been placed on the production cross section due to neutrons. This cross section is $\sim 10^{-2}$ those estimated from the events observed in cosmic-ray emulsion experiments.

The authors wish to thank C.A. Ayre, O. Haas, P.V.R. Murthy and J. Pluta for their help in carrying

$\$ 3$ We estimate that a typical Schein-type event would be accompanied by $\geqslant 2$ electrons in the detector $10 \%$ of the time. out this experiment. We also wish to acknowledge the assistance of H. Haggerty, P. Koehler, J. Sanford, and others of the Fermilab staff.

\section{References}

[1] P.B. Price, E.K. Shirk, W.Z. Osborne and L.S. Pinsky, Phys. Rev. Lett. 35 (1975) 487.

[2] R.A. Carrigan Jr., F.A. Nezrick and B.P. Strauss, Phys. Rev. D 10 (1974) 3867.

[3] L.M. Barkov et al., Soviet Physics JETP 34 (1972) 917.

[4] R.R. Ross et al., Phys. Rev. D8 (1973) 698.

[5] M.A. Ruderman and D. Zwanziger, Phys. Rev. Lett. 22 (1969) 146.

[6] M. Schein, D.M. Haskin and R.G. Glasser, Phys. Rev. 95 (1954) 855; see also, Proc. Rochester Conf. on High energy physics, 1955, p. 117.

[7] A. Debenedetti et al., Nuovo Cimento 12 (1954) 954; A. Debenedetti et al., Nuovo Cimento 2 (1955) 220.

[8] M. Koshiba and M.F. Kaplan, Phys. Rev. 100 (1955) 327

[9] L.B. Silva et al., Nuovo Cimento 3 (1956) 1465.

[10] G.B. Collins et al., Phys. Rev. D8 (1973) 982.

[11] A search for anomalous multigamma events produced by cosmic ray protons was made by the Echo Lake group. See J. Wilkes, Ph.D. thesis; University of Wisconsin, 1974 (unpublished).

[12] For details of the spectrum see: M.J. Longo et al., Characteristics of the M-3 Neutral Beam at NAL, University of Michigan High Energy Group Report UM HE 74-18 (unpublished).

[13] B.W. Loo, Doctoral Thesis, University of Michigan (unpublished).

[14] J.L. Newmeyer and J.S. Trefil, Nuovo Cim. 8A (1972) 703. 